\title{
Origine sociale des religieuses de l'Hôpital-général de Québec (1692-1764)
}

\section{Micheline D’Allaire}

Volume 23, numéro 4, mars 1970

URI : https://id.erudit.org/iderudit/302934ar

DOI : https://doi.org/10.7202/302934ar

Aller au sommaire du numéro

Éditeur(s)

Institut d'histoire de l'Amérique française

ISSN

0035-2357 (imprimé)

1492-1383 (numérique)

Découvrir la revue

Citer cet article

D’Allaire, M. (1970). Origine sociale des religieuses de l'Hôpital-général de Québec (1692-1764). Revue d'histoire de l'Amérique française, 23(4), 559-581. https://doi.org/10.7202/302934ar d'utilisation que vous pouvez consulter en ligne.

https://apropos.erudit.org/fr/usagers/politique-dutilisation/ 


\section{ORIGINE SOCIALE DES RELIGIEUSES DE L'HOPITAL-GÉNÉRAL DE QUÉBEC (1692-1764)}

C'est au problème de la mendicité que se rattache le projet de fonder un Hôpital-Général à Québec. La formule n'est pas neuve, puisqu'en France, l'Etat a déjà institutionnalisé le "renfermement" des pauvres mendiants, en 1656, par la création d'un Hôpital-Général chargé de les faire travailler, chacun selon ses capacités; le succès de l'entreprise amène le Roi, en 1662, à vouloir répéter l'expérience hors de Paris, dans toutes les grandes villes de France. ${ }^{1}$

En Nouvelle-France, c'est Mgr de Laval qui, le premier, pense à la fondation d'un Hôpital-Général, semblable à ceux de France. Supporté dans ses vues par Frontenac et Duchesneau, il craint que la pauvreté, devenue chose courante, finisse par accabler la jeune colonie; en effet, le nombre d'adultes vivant au crochet de l'Etat est de plus en plus grand et trop d'enfants abandonnés s'habituent à la fainéantise et au libertinage. Tous trois, convaincus qu'un Hôpital-Général apprendrait justement aux jeunes à travailler, tombent d'accord pour demander au Roi des lettres patentes autorisant la fondation de cet établissement. ${ }^{2}$

En même temps, Talon, de retour en France depuis 1673, insiste pour établir, à ses propres frais, un Hôpital-Général à Québec, tel que créé en France : il y voit avantage, pour la colonie qui en serait favorisée, tant par l'établissement de manufactures que par l'assistance qu'on pourrait donner aux pauvres. Malheureusement, Dudouyt, représentant de Laval pour les affaires ecclésiastiques à Paris, s'y oppose fortement, ${ }^{3}$ ne voyant, dans les dispositions de Talon, qu'un prétexte pour s'immiscer dans les affaires canadiennes. Les pressions exercées sur la Cour

${ }^{1}$ Louis Merle, "L'Hôpital-Général du Saint-Esprit de Niort (16651790)", Cahiers de la Revue du Bas-Poitou et des Provinces de l'Ouest, $\mathrm{n}^{\circ} 2$ (Fontenay-le-Comte, 1966): 9 .

2 Frontenac, Duchesneau et Laval, ASQ, Polygraphie, XVIII: 45; document sans date, que l'Archiviste du Séminaire situe entre 1675 et 1680 .

3 Dudouyt à Laval, 9 mars 1681 et 9 mai 1681, ASQ, Lettres, N : 52; 26 mai 1682 , ibid.: 62 .

Vol. XXIII, no 4 (mars 1970) 
par Dudouyt ont d'autant plus d'effet que la Cour elle-même s'oppose au projet d'un Hôpital-Général à Québec, craignant, par cette entreprise, de favoriser la fainéantise; du reste, le Roi affirme que la petite population de la colonie ne justifie pas encore la fondation d'un Hôpital-Général : pour l'instant, l'essentiel, en Nouvelle-France, c'est la culture des terres et non l'établissement de manufactures. ${ }^{4}$

D'autre part, au moment où est formé le projet d'un HôpitalGénéral, il existe déjà, dans la colonie, un embryon d'organisation sociale pour parer aux fâcheuses conséquences de l'insécurité publique sans cesse croissante. D'abord, les législations de 1677 et de 1683, en défendant la mendicité dans les villes, ${ }^{5}$ tentent de régler le problème du vagabondage - sans toutefois s'occuper du problème des pauvres mendiants et des vieillards. Mais cette réglementation s'avère bien insuffisante quand les guerres iroquoises recommencent en 1684: d'une part, la colonie connaît une véritable indigence, d'autre part, les habitants en profitent pour mendier. Dès lors, on pense, en 1688, à établir un Bureau des Pauvres à Québec, à Montréal et aux Trois-Rivières; cette institution a pour but de fournir la subsistance aux pauvres et de leur donner du travail, tout en mettant de l'ordre dans la société. ${ }^{6}$ Les caisses de l'entreprise sont alimentées par des marchands influents de Québec, par les communautés religieuses et par des amendes que perçoivent les tribunaux. Toutefois, ce système d'aumônes volontaires n'est pas le moyen financier le plus sûr pour assister les pauvres et pour assurer l'ordre et la sécurité publique, d'une façon efficace.

En 1692, la mendicité a pris une telle ampleur qu'il faut sérieusement penser à modifier l'équipement charitable, devenu inadéquat. Or, pour remédier au mal, certains pensent à utiliser l'Hôtel-Dieu de Québec comme maison de "renfermement" des pauvres; d'autres préfèrent que l'établissement en soit séparé. Mgr de Saint-Vallier est évidemment celui qui se prononce le plus fort en faveur d'un Hôpital-Général distinct de l'Hôtel-Dieu. Il se peut qu'on attribue à l'Evêque différents motifs d'action; par exemple, on peut facilement croire que l'Evêque désire, comme son prédécesseur, Mgr de Laval, qui a fondé le Séminaire

${ }^{4}$ Louis XIV à Champigny, 31 mai 1686, ASQ, Lettres, P: 51.

5 Edits, ordonnances royaux, déclarations et arrêts du Conseil d'Etat du roi concernant le Canada (Québec, E.R. Fréchette, 1854-1856), II: 102.

6 Ibid.: 119; W. J. Eccles, "Social Welfare Measures and Policies in New France", 9-20 du XXXVI Congreso Internacional de Americanistas, (Séville, 1966), vol. 4: 13. 
de Québec, laisser une grande œuvre derrière lui. Quoi qu'il en soit, il reste que le pays a beaucoup évolué depuis Laval : les structures sont maintenant en place, de nouveaux problèmes ont surgi et il faut leur faire face.

Or, le 3 mars 1692, le Roi consent enfin à accorder aux autorités coloniales des lettres patentes permettant l'établissement d'un Hôpital-Général afin de réduire l'oisiveté et développer, chez les jeunes, le goût de l'effort en leur apprenant divers travaux, y compris, bien entendu, la culture de la terre. ${ }^{7}$ La direction de cet Hôpital - qui peut recevoir des mendiants valides et invalides - est composée de l'Evêque, du Gouverneur et de l'Intendant; le curé de Québec et trois laïes en constituent l'administration. Pour gérer les biens des pauvres, il faut trouver des personnes dévouées et capables. L'Evêque propose alors aux religieuses de l'Hôtel-Dieu de donner quatre de leurs sujets au nouvel Hôpital. Malgré de nombreuses objections, les religieuses de l'Hôtel-Dieu, le 9 janvier 1693, acceptent la proposition de l'Evêque, de peur de s'attirer des difficultés dans l'avenir. ${ }^{8} \mathrm{La}$ petite équipe se détache alors de l'Hôtel-Dieu; toutefois, les religieuses, quoique matériellement séparées de leur monastère, demeurent sous la dépendance de celui-ci, en tant que moniales. Cette tutelle dure jusqu'à 1701, année où l'Hôpital-Général se sépare définitivement de l'Hôtel-Dieu pour devenir autonome. Dès lors, l'histoire de l'Hôpital-Général commence vraiment à se dérouler.

Pour l'instant, notre curiosité se porte avant tout sur un aspect social de l'institution: nous nous proposons d'étudier à quel groupe social appartiennent les religieuses de l'HôpitalGénéral.

Sans point de repère, il est impossible de qualifier l'origine sociale des religieuses de l'Hôpital-Général. C'est pourquoi nous avons comparé cette communauté aux deux autres communautés de femmes de Québec, pour la même époque : l'Hôtel-Dieu et le couvent des Ursulines.

Notre principale source d'information vient des divers registres des trois communautés étudiées, de leurs Annales, Actes capitulaires, contrats de dots et livres de comptes. Evidemment, nous avons complété ces données par des recensements du régime

7 Edits et ordonnances, I: 271-274.

8 Annales de l'Hôpital-Général, I: 275. 
français, des pièces notariales et divers travaux de biographie. Tout cela nous a fourni les éléments essentiels sur lesquels repose notre étude du groupe auquel appartiennent les religieuses de l'Hôpital-Général, par comparaison aux deux autres communautés.

L'absence de véritables classes sociales en Nouvelle-France entraîne à des opérations délicates dès qu'on tente de dégager les groupes hybrides qui la constituent. Ce travail requiert d'autant plus de précautions dans l'analyse et de prudence dans les conclusions qu'il est difficile, avant le XVIII ${ }^{\mathrm{e}}$ siècle, de circonscrire les groupes constituants de la colonie : il faut attendre que la population atteigne une certaine consistance, et la société elle-même, des cadres assez stables.

Pour décrire la société de la Nouvelle-France, on est porté, a priori, à la comparer à la société française qui, évidemment, a fait passer bien des habitudes de vie avec les émigrants qu'elle a envoyés au Canada. Cependant, les exigences locales ont peu à peu différencié la société coloniale de la métropolitaine, au point qu'au XVIII ${ }^{\text {e }}$ siècle la France et la Nouvelle-France offrent des phénomènes d'observation différents l'un de l'autre.

$\mathrm{Au}$ vrai, la stratification sociale en France, aux XVII et XVIII ${ }^{e}$ siècles, ne se retrouve pas tout à fait en Nouvelle-France. Ici, point de structures rigides: contrairement à là-bas, c'est la mobilité sociale qui caractérise d'abord la colonie. Par conséquent, il n'existe pas de limites bien claires entre les classes populaires, la bourgeoisie et l'aristocratie. D'ailleurs, comme le dit Cameron Nish, dans une étude récente, "la question de structure de classes en Nouvelle-France n'était tout simplement pas un problème". ${ }^{9}$ Bien sûr, l'existence d'un statut privilégié chez certains individus est indéniable. Mais on ne peut pas pour autant parler de classes: les nobles d'ici ont l'esprit bourgeois; quant à ceux qu'on pourrait appeler "bourgeois", nous les retrouvons, en plus, évidemment, d'être mêlés au commerce, à la fois dans l'administration, dans la vie seigneuriale et dans l'activité militaire. L'homme d'affaires canadien, contrairement au bourgeois français, peut même être placé très haut dans la société: ambitieux, il peut, par ses attitudes et les nouvelles valeurs qu'il adopte, se hisser parmi les nobles, étant donné l'absence de classes bien définies. Aussi, plusieurs riches mar-

${ }^{9} \mathrm{C}$. Nish, Les bourgeois-gentilshommes de la Nouvelle-France (17291748) (Montréal et Paris, Fides, 1968), 183. 
chands d'ici possèdent-ils des lettres de noblesse alors qu'en France, ces personnes seraient considérées comme usurpatrices par la noblesse d'épée. (On sait qu'à partir de 1685 , le noble canadien peut, sans déroger, se livrer au commerce de détail.) La voie utilisée pour devenir noble est généralement celle-ci: on se fait remarquer par les autorités coloniales, puis on devient seigneur; on sait qu'être seigneur est, en soi, une marque distinctive de supériorité sociale. Enfin, on peut, à ce niveau, aspirer aux titres de noblesse. C'est pourquoi l'idée de classes sociales sied mal dans la société française d'Amérique. On aurait peine, par exemple, à reconnaître une classe dominante, en Nouvelle-France, telle que l'historien Mandrou la définit. ${ }^{10}$ Aussi, nous a-t-il paru prudent d'éviter systématiquement l'utilisation du mot classe.

Il ressort, de ces observations, que le travail de compartimentation sociale est infiniment délicat à faire dans cette Nouvelle-France où la conscience de groupe ne se retrouve pas et où l'osmose sociale est facile. Par conséquent, quelle que soit la terminologie utilisée, elle demeure discutable.

Or, tenant compte des difficultés que présente toute tentative de mettre la société en catégories hiérarchisées et de l'état actuel de la recherche en histoire sociale de la Nouvelle-France, nous avons choisi une classification, arbitraire, il est vrai, qui, sans trahir la réalité, évite toute discussion juridico-sociale. Ainsi donc, notre étude n'entend pas établir des critères d'appréciation valables des différentes couches de la société, pas plus qu'elle n'entend analyser leur comportement. Nous en serions d'ailleurs incapable. Eloignant alors les concepts de bourgeoisie et de noblesse (ce qui ne nous empêchera pas de parler de nobles en tant qu'individus dont les titres et qualités révèlent d'une façon patente le caractère de noblesse, les nobles et la noblesse étant deux réalités distinctes), nous avons encadré la population étudiée dans des catégories très larges. En effet, la classification pour laquelle nous avons opté apporte une vision plutôt souple de la société, en la découpant en élite et en masse, plus exactement en dominants et en dominés; cette division vaporeuse permet

$10 \mathrm{R}$. Mandrou attribue à une classe dominante les caractéristiques suivantes: a) pleine conscience et sécurité de moyens; b) genre de vie caractéristique reconnu supérieur à d'autres groupes sociaux; c) conscience que sa culture est la plus riche; d) prise de conscience face au reste de la collectivité. (R. Mandrou, Classes et luttes de classes en France aw début $d u X V I I^{e}$ siècle, Universita Degli Studi di Pisa. Pubblicazioni dell'Instituto di storia della Facolta di Lettere, Casa Editrice G. d'Anna (Messina-Firenze, 1965), 30-32). 
justement de comprendre ce phénomène de capillarité qui attire des individus comme Pierre Boucher et Charles Aubert de La Chesnaye au sommet de la société.

Le mode de répartition de la société entre dominants et dominés est tout aussi valable que d'autres puisqu'en NouvelleFrance il n'existe pas de groupes prééminents multiples comme en France, au XVII ${ }^{\mathrm{e}}$ siècle, où l'on voit, au sommet de la société, la noblesse et la bourgeoisie, formant ensemble "les groupes dominants" dont parlent certains historiens contemporains. Dans la colonie, les personnes qui ont de l'ascendant sur les petites gens forment plutôt un bloc monolithique; par conséquent, n'est perceptible, ici, qu'un seul groupe de dominants ayant, sous sa tutelle, les milieux populaires ou les dominés.

Toutefois, pour découvrir le sommet et la base de la pyramide sociale, nous subdiviserons les dominants en élite de fonction et de dignité, d'une part, et en entrepreneurs, de l'autre. Quant aux dominés, nous les subdiviserons en gens de métier et artisans, et en paysans. Les catégories dans lesquelles nous ferons entrer la population étudiée seront donc les suivantes:
A) élite de fonction et de dignité
B) entrepreneurs
C) gens de métier et artisans
D) paysans

Voilà la méthode qui nous permettra de tracer nos graphiques et, par conséquent, de démontrer les origines sociales des religieuses: étaient-elles recrutées parmi les dominants ou parmi les dominés?

D'abord, nous avons cru nécessaire de qualifier la première catégorie, celle de l'élite, par la fonction et la dignité: cette précision signifie que l'élite de la catégorie A ne comprend qu'une partie de la couche supérieure de la société. Autrement dit, ce regroupement de l'élite de fonction et de dignité n'est qu'une première étape pour découvrir le groupe de l'élite en général, les dominants.

Lorsqu'il s'agissait d'un noble, la classification s'établissait aisément; lorsqu'il s'agissait d'une personne investie d'une fonction importante, comme celle de gouverneur ou de conseiller au Conseil souverain, le regroupement s'opérait également bien. Mais il nous a été parfois difficile d'évaluer l'importance de la fonction qu'il exerçait comme écrivain. Toutefois, malgré les 
hésitations et même les risques, nous pouvons, dans l'ensemble, arriver à des résultats plausibles.

La catégorie suivante, celle des entrepreneurs, ne comprend pas nécessairement des personnes fortunées, puisqu'on ne sait pas qui était riche, en Nouvelle-France: on pouvait l'être pendant cinq ans et devenir pauvre par la suite. L'exemple de La Chesnaye est typique. Par ailleurs, l'ensemble de nos entrepreneurs n'offre pas un aspect quantitatif suffisant pour les diviser en élite riche et en élite moins riche. Sans compter que le montant de la dot pouvait bien nous donner un indice de l'état de fortune au moment même de la dotation, mais c'était insuffisant pour amener à conclure sur la valeur de tel entrepreneur en particulier. La catégorie $B$ ne vise donc pas à donner une image réelle du milieu des affaires; c'est pourquoi nous avons choisi le terme très large de "entrepreneur", terme qui comprend ici les commerçants médiocres comme les gros marchands. La caractéristique commune des personnes qui en font partie, c'est qu'elles agissent dans la société en participant d'une façon quelconque aux affaires. Un entrepreneur, à notre sens, c'est celui qui effectue, par lui-même ou par l'intermédiaire d'employeurs, un travail rémunérateur, c'est celui qui dirige une entreprise quelconque en acceptant les risques et en profitant des bénéfices.

Quant aux dominés, ils comprennent les gens de métier, les artisans et les paysans. Par paysans, nous entendons les personnes qui habitent à la campagne et qui cultivent la terre. L'épithète de paysan n'est évidemment indiquée nulle part dans les divers registres ou contrats; celle de cultivateur ne l'est pas toujours, loin de là. Par le biais, nous sommes tout de même arrivée à savoir qui était paysan, d'après le lieu d'origine, la dot ou le genre de procès dans lesquels étaient impliqués certains pères de religieuses. Il est bien entendu qu'un cultivateur riche et entrepreneur, par hypothèse celui qui aurait plus de 100 arpents en valeur, a été placé dans la catégorie B et non D.

Malgré les difficultés encourues, nous avons pu, dans l'ensemble, arriver à des résultats plausibles, résultats qui nous ont ensuite amenée à l'étude des dots, indispensable complément à celle de l'origine sociale, puisque, dans notre hypothèse de base, nous avons supposé une certaine corrélation entre le niveau social des familles et leur état de fortune; nous nous sommes demandé si la dignité et la richesse étaient des attributs jumeaux.

Or, pour découvrir le lien possible entre la situation financière et la condition sociale des familles de religieuses, nous 
avons essayé de connaître la valeur de la dot donnée par chaque professe. Encore ici, nous avons établi une comparaison entre les trois communautés de femmes de Québec. Quant à la méthode suivie, elle dépend, en partie, de la disponibilité des documents. Nous avons déjà parlé des sources essentielles sur lesquelles repose notre étude des dots: les registres des communautés, les Actes capitulaires, les contrats de dots ou de profession, les Annales et les livres de comptes.

La première étape de l'étude a pour but de comparer les différents groupes sociaux représentés à l'intérieur de chaque communauté. Nous avons alors découpé la période 1693-1764 en décennies et, pour chacune, avons rassemblé le nombre de pères de religieuses appartenant à chaque groupe social.

Nous constatons que $35.5 \%$ des religieuses de l'HôpitalGénéral sont recrutées parmi l'élite de fonction et de dignité, comparé à $22 \%$ des religieuses de l'Hôtel-Dieu et $21.4 \%$ des Ursulines, pour la même catégorie: la supériorité revient nettement aux religieuses de l'Hôpital-Général.

Nos calculs nous ont d'abord permis d'observer qu'à l'Hôpital-Général, le nombre de nouvelles recrues appartenant à la catégorie $A$ (élite) suit une évolution assez normale ${ }^{11}$ une fois la période d'établissement achevée - période correspondant au grand besoin de converses et à l'absence prolongée de SaintVallier - l'augmentation se fait brusquement au cours des années 1713-1722, où l'on passe d'une recrue à dix; le retour de l'évêque, en 1713, aide sans doute au recrutement dans les paroisses, sans compter que la construction de deux ailes à l'Hôpital et l'ouverture d'une maison pour aliénées mentales, en 1717 , demandent un plus grand nombre de religieuses pour assumer ces nouvelles responsabilités.

A partir de 1734, on accueille à nouveau des postulantes; cette même année, la Cour permet à la Communauté de recevoir des militaires hors d'état de faire leur service, ce qui exige un surplus de religieuses. Nous voyons alors le nombre de religieuses, originaires de l'élite de fonction et de dignité, se maintenir au même niveau qu'il était au cours de la période 1713-1722. Le nombre de sujets diminue à neuf au cours des années de

11 Si l'on soustrait, évidemment, la période 1723-1732 où, à cause de la surveillance rigoureuse des stipulations relatives à la dot, portée alors à 5,000 livres au lieu de 3,000 , aucune religieuse n'entre au monastère. 
guerre (1743-1752), fléchissement qui s'accentue pendant la dernière décennie jusqu'à six recrues : plusieurs filles de l'élite disparaissent.

Nous avons effectué nos calculs de la même façon pour ce qui est de l'Hôtel-Dieu. Comparée aux trois communautés, celle de l'Hôtel-Dieu se place au deuxième rang dans la catégorie A (élite), avec un décalage de $13.5 \%$ sur l'Hôpital-Général. Expliquons brièvement comment ce pourcentage se distribue sur la période 1693-1764.

Le nombre de filles issues de familles de l'élite varie considérablement à l'Hôtel-Dieu au cours de la période 1693-1764. Pendant la décennie 1703-1712, les effectifs doublent par rapport à la période précédente, passant de quatre recrues à neuf. Nous faisons remarquer que ces années se situent immédiatement après le conflit survenu entre l'Hôtel-Dieu et l'Hôpital-Général au sujet de la séparation des deux maisons (1699-1701). Nous sommes portée à croire que cette querelle retentissante incite les filles de l'élite à entrer dans une maison plutôt que dans une autre. Mais le nombre redescend rapidement à trois au cours des années 1713-1722 et à zéro pendant la décennie 1723-1732. La diminution de recrues, en général, s'explique par les mêmes raisons qui ont amené la fermeture du noviciat de l'HôpitalGénéral de 1720 à 1734 . Après un dernier sursaut à six recrues de 1733 à 1742, années où les nouvelles religieuses originaires de la catégorie A (élite) enregistrent la moitié des entrées de la décennie, il y a diminution jusqu'à la fin de la période où cette catégorie A (élite) ne compte aucune entrée pendant les onze dernières années.

De leur côté, les Ursulines comptent, pour l'ensemble de la période, $21.4 \%$ de leur population recrutée parmi ce groupe de l'élite de fonction et de dignité. Comme à l'Hôtel-Dieu, la distinction s'y fait d'une façon accidentée tout au long de la période; jusqu'à la décennie 1723-1732, il y a augmentation constante et accentuée: dès les dix premières années, de 1693 à 1702, le monastère des Ursulines enregistre l'entrée de cinq filles appartenant à l'élite - chiffre élevé par comparaison avec les autres groupes sociaux - ; ce nombre est ensuite porté à neuf pendant la décennie 1713-1722, pour redescendre à deux seulement de 1723 à 1732 (période où les recrues sont d'ailleurs peu nombreuses en général); de 1723 à 1742, il n'y a aucune entrée de filles appartenant à la catégorie A (élite); la Communauté n'en comptera ensuite que deux de 1743 à 1752 , et une seule de 1753 à 1764 . 
Passant du groupe A (élite) au groupe B (entrepreneurs), l'ordre des priorités se trouve inversé: les Ursulines ont maintenant la supériorité avec $25.2 \%$ de leur population appartenant au groupe des entrepreneurs; l'Hôtel-Dieu en compte $16.5 \%$ et l'Hôpital-Général, $15.9 \%$.

Ce qui frappe chez les Ursulines, c'est l'entrée constante et progressive de filles d'entrepreneurs dans la Communauté. Nous remarquons, par exemple, qu'au cours des dix années 1723-1732, alors que peu de nouveaux sujets entrent au monastère, le nombre de filles d'entrepreneurs prend la vedette, représentant quatre recrues. Puis, pendant la décennie 1743-1752, le monastère accueille un plus grand nombre de sujets: si l'on excepte les religieuses d'origine inconnue, la moitié des nouvelles recrues, soit huit, viennent du groupe des entrepreneurs.

Vient au deuxième rang de la catégorie des entrepreneurs, l'Hôtel-Dieu. Au cours de la période 1693-1764, on y enregistre deux sommets, quoique peu élevés, étant donné, en général, dans les trois communautés, le nombre inférieur des sujets appartenant à ce groupe. Le plus haut sommet se maintient au niveau de quatre recrues pour chaque décennie, de 1703 à 1712 et de 1713 à 1722. Puis le nombre tombe à une recrue de 1723 à 1732 pour ensuite descendre à zéro de 1733 à 1742 ; le nombre augmente à nouveau à trois recrues pendant chacune des décennies 1743-1752 et $1753-1764$.

Pour la période 1693-1764, le groupe de filles d'entrepreneurs à l'Hôpital-Général compte la moitié moins de recrues que le groupe des filles de l'élite de fonction et de dignité. De 1693 à 1702, on compte trois filles d'entrepreneurs sur neuf nouvelles recrues. Au cours des dix ans qui suivent, 1703-1712, on n'en comptera qu'une seule sur neuf, également. Après cette période de tâtonnement qui correspond au début de l'établissement, on remarque une augmentation subite des effectifs de la Communauté en général: on passe de neuf recrues (1703-1712) à 22 recrues (1713-1722). On note alors une légère augmentation du nombre de religieuses issues de familles d'entrepreneurs: on passe de trois à quatre. Puis, lorsqu'on recommence à accueillir des postulantes, en 1734, le nombre de filles d'entrepreneurs monte un peu pour égaler la moitié du nombre de l'élite qui comptait dix recrues. Mais le nombre des filles d'entrepreneurs à se présenter au monastère diminue à trois pendant la décennie 1743-1752; cependant, quatre nouvelles recrues dont l'origine demeure inconnue, peuvent ici faire varier le nombre. 
A la fin de la période, il n'y a plus qu'une seule fille d'entrepreneur qui se présente à l'Hôpital-Général, de 1753 à 1764 .

S'agit-il du nombre de religieuses, filles d'hommes de métier ou d'artisans, l'Hôtel-Dieu prend la tête des trois communautés: elle en enregistre $24.7 \%$ de sa population, alors que l'HôpitalGénéral se situe au deuxième rang avec $16.8 \%$ et la communauté des Ursulines au dernier rang, avec le faible pourcentage de $9.7 \%$.

La distribution des entrées de filles d'artisans à l'Hôtel-Dieu semble se faire un peu au hasard. Nous avons remarqué une montée très forte de filles d'artisans de 1703 à 1722: de trois qu'elles étaient pendant la décennie précédente, elles sont maintenant dix; cette montée devait se modifier en une descente abrupte par la suite, en nombre plus qu'en pourcentage, cependant: ce groupe passe à quatre recrues (1713-1722), à deux (1723-1732), puis à une seule (1733-1742), pour ensuite remonter à trois (1743-1752), puis à quatre nouvelles recrues pendant la dernière décennie.

A l'Hôpital-Général, on constate un accroissement, constant mais léger, du nombre de filles d'artisans. Pendant les dix premières années, on n'enregistre aucune recrue appartenant à cette catégorie $\mathrm{C}$ (artisans). Au cours de la période 1703-1712, deux se présentent au monastère, nombre qui se maintient de 1713 à 1722. Puis, de 1734 à 1742, quatre recrues, filles d'artisans, y entrent. L'Hôpital-Général en accueille cinq au cours de 1743 à 1752; le même nombre s'y présente de 1753 à 1764 .

Ce qui frappe d'abord, chez les Ursulines, c'est le petit nombre de filles d'artisans. La première entre au cours de la période 1703-1712; deux autres s'y ajoutent de 1713 à 1722 . Il faut ensuite attendre la décennie 1733-1742 pour y voir accueillis trois autres sujets. Quatre seulement se présenteront au monastère jusqu'à la fin: trois de 1743 à 1752 et une de 1753 à 1764 .

Le groupe $\mathrm{D}$, celui des paysans, est plus élevé, dans les trois communautés, par rapport au groupe précédent. L'Hôtel-Dieu occupe encore la première place avec une population qui se compose de $35.8 \%$ de filles de paysans, tandis que la communauté des Ursulines en compte $28.2 \%$ et celle de l'Hôpital-Général, $24.3 \%$.

Pour l'ensemble de la période 1693-1764, nous constatons, à l'Hôtel-Dieu, un nombre plutôt élevé de filles de paysans, soit 
39 recrues, même si ce chiffre subit une chute après 1713 , passant de huit (1713-1722), à quatre (1723-1732); chacune des décennies 1733-1742 et 1743-1752 en compte cinq; enfin, trois sont enregistrées pendant les dix dernières années. Si nous tenons compte de la faible population de la Communauté, vers la fin de la période, nous constatons que le nombre de filles de paysans, quoique diminué, demeure assez fort relativement au nombre de celles qui viennent des autres groupes sociaux. Soulignons que l'entrée de nombreuses converses contribue à donner de l'importance au groupe des paysans puisque la très grande majorité de ces religieuses sont filles de cultivateurs.

Chez les Ursulines, également, ce sont les converses qui forment la population d'origine paysanne: trois filles de paysans entrent pendant la décennie 1693-1702 et quatre, de 1703 à 1712 . Ce groupe double ses effectifs de 1713 à 1722 pour monter à huit nouvelles recrues; lors de la période difficile de 1723 à 1732, on ne compte que deux nouveaux sujets appartenant à la catégorie des paysans sur un total de dix recrues; le nombre augmente à trois de 1733 à 1742 , puis à cinq de 1743 à 1752 , pour redescendre à quatre pendant les dix dernières années.

A l'Hôpital-Général, nous constatons que le nombre de filles de paysans - quoique assez élevé, dans l'ensemble de la période 1693-1764, relativement aux autres groupes sociaux - forme, par rapport aux autres communautés, le groupe dont le pourcentage est le moins élevé. Il est intéressant de remarquer que, contrairement à la catégorie A (élite), le groupe des paysans est élevé au début et à la fin de la période. Que les paysans soient en plus grand nombre au début - cinq sur neuf de 1693 à 1702 et quatre de 1703 à 1712 -, ce nombre n'est pas étonnant car, au cours de ces années, le quart de la Communauté est constitué de converses, sujets sans doute très utiles en période d'établissement. Le peu d'exigence de la Communauté concernant les dots, a, par ailleurs, l'effet d'attirer les filles de familles pauvres. Au cours des années 1713-1722, les filles de paysans augmentent en nombre (six nouvelles recrues), mais baissent par rapport à la moyenne des autres groupes. Après la réouverture du monastère, en 1734 , on voit baisser le nombre de filles de paysans à quatre recrues sur un total de 23 pour tomber à deux seulement pendant la décennie 1743-1752. Ici, cependant, les quatre religieuses dont l'origine demeure inconnue peuvent légèrement faire varier le nombre des différents groupes. Enfin, le groupe des paysans reprend de l'importance lors de la dernière décennie pour monter à cinq nouvelles recrues. 
Un des objectifs de notre étude étant de comparer la population des trois communautés, voici maintenant le pourcentage des différents groupes sociaux par rapport à l'ensemble des communautés.

Les trois communautés comptent 84 religieuses appartenant au groupe de l'élite de fonction et de dignité, sur un total de 319 (dont 25 d'origine inconnue). Nous constatons qu'avec $45.2 \%$ des 84 religieuses, le pourcentage de la communauté de l'HôpitalGénéral dépasse celui de l'Hôtel-Dieu de 16.3 et celui de la communauté des Ursulines de 19. Cependant, l'Hôpital-Général baisse en nombre et en importance par rapport aux deux autres communautés quand nous considérons la somme des filles d'entrepreneurs: leur nombre à l'Hôpital-Général s'élève à $27.9 \%$ de l'ensemble, à l'Hôtel-Dieu, à $29.5 \%$ et, à la communauté des Ursulines, à $42.6 \%$. Remarquons que l'ensemble des trois communautés compte peu de sujets faisant partie du groupe des entrepreneurs: 61 religieuses réparties dans les trois communautés. Le groupe $\mathrm{C}$ (hommes de métier et artisans) en compte encore moins: 55 seulement. Il va sans dire que l'interprétation des pourcentages de distribution exige beaucoup de prudence. Cette fois, au groupe C (artisans), c'est la communauté de l'Hôtel-Dieu qui domine avec un pourcentage de $49.1 \%$; celle de l'Hôpital-Général suit avec $32.7 \%$ et celle des Ursulines se situe en dernier avec 18.2\%. Le groupe D (paysans) fournit un élément de comparaison plus intéressant que les autres, étant donnée une certaine consistance de population qui le constitue: 96 personnes pour l'ensemble des communautés. C'est encore l'Hôtel-Dieu qui se coiffe du plus haut pourcentage: $41.5 \%$ de l'ensemble des filles de paysans connues; la communauté des Ursulines se place au deuxième rang avec $30.9 \%$; suit celle de l'Hôpital-Général avec $27.7 \%$. Le nombre des inconnues, enfin, n'est pas dépourvu de signification, surtout en ce qui concerne la communauté des Ursulines. Que cette dernière communauté enregistre $64 \%$ de l'ensemble des religieuses dont l'origine demeure inconnue, cela peut sembler énorme; en réalité, c'est par comparaison avec l'Hôpital-Général qui compte huit inconnues seulement et celle de l'Hôtel-Dieu qui n'en compte qu'une seule. Ces observations laissent déjà deviner vers quel monastère s'orientaient surtout les jeunes filles de la colonie recrutées dans un groupe ou dans l'autre de la société.

Pour illustrer, d'une façon plus nette, la physionomie sociale de chaque communauté, nous avons ensuite groupé, sous le nom de "dominants", les religieuses recrutées parmi l'élite de fonction 
et de dignité et parmi les familles d'entrepreneurs, et sous le nom de "dominés", les religieuses dont les pères sont soit artisans soit hommes de métier soit paysans. Nous illustrons ici le pourcentage de dominants et de dominés, en ayant soin de soustraire, pour chacune des trois communautés, le nombre de postulantes sorties.

Pour l'élément dominant, l'Hôpital-Général l'emporte avec $59.8 \%$ de sa propre population originaire de ce groupe social. Du côté des dominés, la supériorité revient à la communauté de l'Hôtel-Dieu qui enregistre un pourcentage élevé de $60.6 \%$ par rapport à sa propre population. La communauté des Ursulines, relativement à sa population, rassemble $51.8 \%$ d'éléments dominants et $43.4 \%$ de dominés.

Selon nos résultats, l'Hôpital-Général serait la Communauté qui posséderait le plus d'éléments dominants - relativement aux trois communautés groupées - pour l'ensemble de la période 1693-1764: $36.6 \%$ de la population globale des dominants. Suivrait la communauté des Ursulines avec un pourcentage de $32.1 \%$ et celle de l'Hôtel-Dieu avec $31.3 \%$. Le groupe des dominés accuse des différences plus grandes encore: à l'Hôtel-Dieu, il s'élève à $48.9 \%$ de l'ensemble, à la communauté des Ursulines à $26.7 \%$ et, à l'Hôpital-Général à $24.4 \%$.

Telle est, en termes plutôt arides, l'approximation valable que nous voulions atteindre. Toutefois, avant de tirer quelques explications et conclusions sur les origines sociales des religieuses, essayons de découvrir, par une brève étude des dots, s'il existe une certaine relation entre le niveau social des familles dominantes et la somme de biens matériels possédés.

Notre étude qui, encore ici, s'est portée successivement sur chacune des trois communautés de femmes, a d'abord tenté d'établir, par décennies, une moyenne de dot par religieuse de chœur et par converse; puis le calcul de la moyenne des dots s'est ensuite fait par rapport à l'ensemble de la période 1693-1764. Il nous a ensuite été possible de dresser un tableau comparatif des trois communautés.

La moyenne des dots des religieuses de chœur pendant la première décennie est la seule du tableau qui, pour l'instant, attire notre attention par sa somme peu élevée. Il faut savoir que, parmi les cinq religieuses de l'Hôtel-Dieu qui viennent s'établir à l'Hôpital-Général, une seule y apporte sa dot; les autres la laissent à leur ancien monastère. Par ailleurs, la reli- 
Moyenne des dots par décennies : l'Hôpital-Général de Québec

\begin{tabular}{cccccc}
\hline Années & $\begin{array}{c}\text { Nombre de } \\
\text { religieuses } \\
\text { de chœur }\end{array}$ & Moyenne & $\begin{array}{c}\text { Nombre de } \\
\text { converses } 13\end{array}$ & Moyenne \\
\hline $1693-1702$ & 4 & 575 livres & - & - \\
$1703-1712$ & 6 & $1,188.5$ & “ & - & - \\
$1713-1722$ & 12 & $1,650.7$ “ & 6 & 336.7 livres \\
$1723-1732$ & - & - & - & - \\
$1733-1742$ & 10 & 2,490 & “ & 5 & 470 “ \\
$1743-1752$ & 15 & 2,850 & “ & 2 & 500 “ \\
$1753-1764$ & 8 & $3,388.9$ & “ & 4 & 137.5 “ \\
\hline
\end{tabular}

gieuse Angélique Hayot n'apporte qu'une dot de 300 livres, ayant obtenu le privilège d'être reçue en l'honneur de saint Joseph. Cette prérogative, inscrite dans les Constitutions de l'HôpitalGénéral, permet à la Communauté de recevoir quelques sujets sans dot en l'honneur de la Sainte Vierge ou de saint Joseph. Outre ce privilège, dont on n'abuse pas d'ailleurs, plusieurs religieuses deviennent professes grâce à Saint-Vallier qui donne, soit des dots entières, soit des dots partielles, soit encore des parties de dots de fondation (contrat de donation par lequel SaintVallier affecte 1,100 livres de rentes annuelles pour servir, à perpétuité, à l'entretien de religieuses).

L'étude des dots - promises ou données - de l'HôpitalGénéral indique, par leur nature, le peu d'argent qu'il y a dans la colonie. Certains parents, par exemple, proposent pour dot de leur fille, des contrats de rentes, soit sur des terrains, soit sur des maisons, ne pouvant donner qu'une partie de la dot, au comptant, ou rien du tout.

Que de religieuses offrent des terres comme dot ! Et combien de pères vantards sont assez habiles à séduire la Communauté en faisant miroiter la bonté d'une terre dont la valeur réelle décevra les religieuses quand elles voudront la revendre quelques années plus tard!

${ }^{12}$ Ce nombre ne représente que les religieuses dont nous connaissions, d'une façon assez certaine, le montant de la dot. Cette remarque vaut pour les deux tableaux suivants.

13 Voir note précédente. 
La dot en terre n'est pas la plus singulière qui soit: des pères paient parfois avec des planches, des madriers, des rentes viagères; on complète encore des dots par des dons provenant tantôt de communautés religieuses, tantôt du Gouverneur. Voilà autant de moyens de suppléance pour tirer d'embarras certains pères incapables d'offrir des dots complètes à leur fille religieuse. Le nombre de filles de familles dominantes en peine d'argent est d'ailleurs un fait qui a attiré notre attention; par exemple, les filles de Charles Damours de Louvières et d'Aubert de La Chesnaye sont en peine: la première doit quêter sa dot, la deuxième, compter sur la dot de fondation.

Comparons maintenant cette application du système des dots aux autres communautés de femmes de Québec, pour la même période, en illustrant d'abord la moyenne des dots des religieuses de l'Hôtel-Dieu.

Moyenne des dots par décennies: l'Hôtel-Dieu de Québec

\begin{tabular}{ccccccc}
\hline Années & $\begin{array}{c}\text { Nombre de } \\
\text { religieuses } \\
\text { de chœur }\end{array}$ & Moyenne & $\begin{array}{c}\text { Nombre de } \\
\text { converses }\end{array}$ & Moyenne \\
\hline $1693-1702$ & 13 & $2,215.4$ livres & 4 & 475 & livres \\
$1703-1712$ & 25 & 1,694 “ & 5 & 480 & “ \\
$1713-1722$ & 16 & $2,793.8$ “ & 3 & 633.3 “ \\
$1723-1732$ & 1 & 4,000 & “ & 5 & 540 & “ \\
$1733-1742$ & 7 & $2,885.7$ “ & 4 & 625 & . \\
$1743-1752$ & 10 & 2,195 & $\cdots$ & 3 & 366.7 & “ \\
$1753-1764$ & 7 & $1,926.1$ & & 2 & 150 & “ \\
\hline
\end{tabular}

Comme à l'Hôpital-Général, la communauté de l'Hôtel-Diea reçoit à la profession quelques religieuses sans dot; des religieuses sont acceptées gratuitement pour services rendus par leur père; d'autres sont acceptées à cause d'une parente qui serait morte très jeune dans la Communauté et aurait elle-même apporté sa dot. Par considération pour la Supérieure, il arrive aussi qu'on diminue la dot. Enfin, le chapitre de la Communauté a parfois des égards bienveillants envers certaines religieuses converses.

A l'Hôtel-Dieu, plus qu'ailleurs, le manque d'argent comptant dans la colonie est manifesté par l'abondance de rentes données 
en guise de dots des futures professes. Outre les constitutions de rentes, il arrive qu'on ramasse tous ses biens pour en faire une donation entière à la Communauté. Mais la donation de terres est encore plus fréquente. Quant aux dots reçues en monnaie de cartes - qu'on se hâte toujours d'utiliser dès la réception - elles semblent moins populaires à l'Hôtel-Dieu qu'à l'HôpitalGénéral. Toutefois, dans l'ensemble, la nature d'un bon nombre de dots est une véritable manifestation du commerce de troc qui se fait dans la colonie: des barriques de vin, de la filasse, du linge, des rideaux, des meubles, du bois, un hangar construit par un père, un métier (celui de la cordonnerie, par exemple), du blé et d'autres denrées.

Enfin, que donne l'étude des dots au couvent des Ursulines ?

Moyenne des dots par décennies: les Ursulines de Québec

\begin{tabular}{ccccccc}
\hline Années & $\begin{array}{c}\text { Nombre de } \\
\text { religieuses } \\
\text { de chœur }\end{array}$ & Moyenne & $\begin{array}{c}\text { Nombre de } \\
\text { converses }\end{array}$ & Moyenne \\
\hline $1693-1702$ & 7 & $2,414.3$ livres & 4 & 350 livres \\
$1703-1712$ & 5 & 2,400 & “ & 3 & 716.7 “ \\
$1713-1722$ & 18 & $2,944.4$ “ & 4 & 575 & “ \\
$1723-1732$ & 5 & 4,000 & “ & 2 & 550 & “ \\
$1733-1742$ & 6 & $2,583.3$ & “ & 3 & 396.7 “ \\
$1743-1752$ & 12 & $2,008.3$ “ & 5 & 500 & “ \\
$1753-1764$ & 5 & 2,100 & “ & 2 & 350 & “ \\
\hline
\end{tabular}

Chez les Ursulines, comme dans les deux autres institutions, plusieurs postulantes offrent des terres comme dot ou partie de dot; cependant, ce genre de donation est plus courant chez les converses. On observe aussi que plusieurs dots sont constituées en cartes. Dans l'ensemble, nous constatons qu'en dépit du statut social important de plusieurs Ursulines, on n'arrive pas toujours à donner la dot ordinaire. Toutefois, cette communauté semble un peu plus en mesure d'assumer les frais d'entretien de certains sujets dépourvus de biens.

L'étude comparative des dots des trois communautés de femmes de Québec ne nous a pas apporté les résultats espérés: trop de documents vagues et imprécis nous tiennent loin de la 
réalité. Notre recherche ne fournit qu'une certaine indication sur l'état de fortune des familles des religieuses et, encore là, l'information ne vaut que pour le moment où la dot est donnée. Malgré tout, le parallèle, dont voici le tableau final, n'est pas dépourvu de signification.

Tableau comparatif des moyennes des dots par religieuse

\begin{tabular}{|c|c|c|c|c|c|}
\hline & & Rel. de cl & œur & Conv & verses \\
\hline $1693-1702$ & Hôpital-Général & 575 liv & res & & - \\
\hline & Hôtel-Dieu & $2,215.4$ & “ & 475 & livres \\
\hline & Ursulines & $2,414.3$ & “ & 350 & “ \\
\hline $1703-1712$ & Hôpital-Général & $1,188.5$ & “ & & - \\
\hline & Hôtel-Dieu & 1,694 & “ & 480 & $“$ \\
\hline & Ursulines & 2,400 & “ & 716.7 & “ \\
\hline $1713-1722$ & Hôpital-Général & $1,650.7$ & “ & 336.7 & “ \\
\hline & Hôtel-Dieu & $2,793.8$ & “ & 633.3 & “ \\
\hline & Ursulines & $2,944.4$ & “ & 575 & “ \\
\hline $1723-1732$ & Hôpital-Général & 一 & & & - \\
\hline & Hôtel-Dieu & - & & 540 & “ \\
\hline & Ursulines & 4,000 & “ & 550 & “ \\
\hline $1733-1742$ & Hôpital-Général & 2,490 & “ & 470 & “ \\
\hline & Hôtel-Dieu & $2,885.7$ & “ & 625 & “ \\
\hline & Ursulines & $2,583.3$ & “ & 396.7 & “ \\
\hline $1743-1752$ & Hôpital-Général & 2,850 & “ & 500 & “ \\
\hline & Hôtel-Dieu & 2,195 & “ & 366.7 & “ \\
\hline & Ursulines & $2,008.3$ & “ & 500 & “ \\
\hline $1753-1764$ & Hôpital-Général & $3,388.9$ & “ & 137.5 & “ \\
\hline & Hôtel-Dieu & $1,926.1$ & “ & 150 & “ \\
\hline & Ursulines & 2,100 & “ & 350 & “ \\
\hline
\end{tabular}

Pour approximatifs qu'ils soient, ces chiffres sont significatifs. Ils montrent d'abord que la moyenne des dots de l'HôpitalGénéral est inférieure à celles des deux autres institutions depuis la décennie 1693-1702 jusqu'à la décennie 1743-1752, alors qu'elle l'emporte remarquablement pendant les vingt dernières années. Nous aurions peine à fournir les raisons qui conditionnent cette infériorité du début si nous n'observions, en même temps, la 
progression constante de la moyenne des dots depuis la fondation jusqu'à la fin du régime français. Tout nous apparaît alors normal quand on songe qu'au moment de la fondation de l'HôpitalGénéral les deux autres institutions existent depuis déjà 53 ans. Par conséquent, il y a déjà une tradition établie dans ces anciennes maisons, ce qui peut attirer là, plutôt qu'à la nouvelle, les filles de familles fortunées. Bien sûr, l'explication demeure partielle. Nous observons, d'autre part, que le bond se fait surtout sentir à partir des années 1733-1742: l'ouverture du pensionnat en $1725 \mathrm{y}$ a-t-il déjà fourni des sujets plus fortunés que ne l'ont été les postulantes d'avant 1720 ? Les troubles causés par la mort de Saint-Vallier - et toutes les factions qui se sont créées dans la colonie - ont-ils discrédité le monastère de l'Hôtel-Dieu au profit de l'Hôpital-Général ? Autant de questions auxquelles nous ne pouvons pas répondre. Chose certaine, l'état général des finances commence à se rétablir.

Concernant l'Hôtel-Dieu, le tableau de la moyenne des dots (p. 576) enregistre des variations d'une décennie à l'autre; nous remarquons notamment que les deux dernières décennies présentent, contrairement à l'Hôpital-Général, une diminution prononcée. Comme pour l'Hôtel-Dieu, nous voyons, chez les Ursulines, une diminution de la valeur des dots à partir de la décennie 1733-1742.

Pour l'ensemble de la période 1693-1764, les moyennes totales des dots des trois communautés se présentent comme suit:

\begin{tabular}{lll} 
& Rel. de chœur & Converses \\
\hline Hôpital-Général & $2,170.7$ livres & 358.8 livres \\
Hôtel-Dieu & $2,221.3$ “ & 492.3 “ \\
Ursulines & $2,576.4$ “ & 493.1 “ \\
\hline
\end{tabular}

La différence entre la moyenne de l'Hôpital-Général et celle de l'Hôtel-Dieu, pour les religieuses de chœur, est de 50.6 livres. Ce montant n'est pas énorme comparé à la différence qui existe entre l'Hôpital-Général et la communauté des Ursulines: 405.7 livres.

Il est intéressant de connaître la moyenne approximative des dots provenant des sujets recrutés dans la classe dominante de la société. Encore une fois, les religieuses ursulines se placent en tête avec une moyenne de 2,653.7 livres par professe de chœur ; 
l'Hôtel-Dieu suit avec 2,500 livres et l'Hôpital-Général avec 2,310 livres; l'ordre demeure donc le même, mais le décalage est moins grand.

Les lacunes de la documentation et le caractère restreint du groupe de personnes étudiées sur une si longue période (environ 300 personnes si l'on excepte les religieuses dont l'origine est inconnue) nous incitent à user de précautions dans les conclusions à tirer sur les origines sociales des religieuses. Cette réserve étant faite, il demeure que nous sommes arrivée à un ensemble de résultats dont le poids n'est pas négligeable: nous pouvons maintenant présenter une certaine image de la communauté de l'Hôpital-Général pendant la période 1693-1764.

Nous constatons d'abord que l'origine sociale des religieuses est de qualité supérieure, dans l'ensemble de la période, aux deux autres communautés. L'Hôpital-Général, en effet, est la communauté qui compte le plus de sujets appartenant à la partie dominante de la société, soit $59.8 \%$ relativement à la population de la Communauté elle-même, sans compter les religieuses sorties. Par rapport à l'ensemble des trois communautés, son pourcentage baisse, évidemment, mais il garde sa supériorité: sans compter les postulantes sorties, l'Hôpital-Général enregistre $36.6 \%$ de toutes les religieuses originaires du groupe dominant de la société. Soulignons, cependant, que le caractère dominant de l'Hôpital-Général vient du grand nombre de sujets recrutés parmi l'élite de fonction et de dignité beaucoup plus que parmi les filles d'entrepreneurs.

Deux conclusions s'imposent: il y a nette évolution sociale chez les religieuses de l'Hôpital-Général de 1692 à 1764; l'origine sociale des religieuses de l'Hôpital-Général est, dans l'ensemble de la période, supérieure à celle des communautés de l'Hôtel-Dieu et des Ursulines. Les résultats de la recherche indiquent même qu'une partie de la Communauté est issue de familles dont le chef est noble. Si, par exemple, nous comptons le nombre de religieuses dont le père est chevalier de saint Louis (pour devenir chevalier de saint Louis, il fallait être noble), la communauté de l'Hôpital-Général compte, pour l'ensemble de la période 1693-1764, 16 filles de chevaliers de saint Louis, alors que celle des Ursulines n'en compte que sept et celle de l'Hôtel-Dieu, qu'une seule. Quant au nombre de filles d'écuyers, c'est-à-dire de père noble, il est également significatif, car, même en supposant qu'ils ne sont pas tous des nobles authentiques, la proportion de faux écuyers devrait normalement être partagée dans les 
trois communautés. Or la communauté de l'Hôpital-Général compte 22 écuyers, tandis que celles de l'Hôtel-Dieu et des Ursulines en comptent respectivement neuf et trois. Enfin, les religieuses, filles de nobles militaires, sont, à l'Hôpital-Général, au nombre de 15, chez les Ursulines, de treize et à l'Hôtel-Dieu, de cinq. ${ }^{14}$

Quand on songe à tout le renoncement et au dévouement que le soin des malades, des vieillards, des invalides, des insensés, des femmes de mauvaise vie - en somme toute une population de condition très humble - exige des religieuses de l'HôpitalGénéral, on est surpris de constater qu'une partie si importante de la Communauté vient de l'élite de fonction et de dignité de la société. Y sont-elles attirées par l'ambiance spirituelle qui règne au monastère ? ou par le désir de la sainte abjection ? En effet, c'est l'époque où l'on voit des âmes en quête de l'humilité la plus parfaite. En outre, la présence de Saint-Vallier, pendant plus de 15 ans à l'Hôpital-Général, ne contribue-t-elle pas à la popularité de la jeune Communauté ? Effectivement, il se fait luimême l'exemple vivant du plus grand renoncement. Par ailleurs, il faut songer que l'Evêque est bien placé pour faire du recrutement auprès des curés et peut-être, aussi, parmi les dominants de la société. D'autres facteurs plus matériels peuvent aussi contribuer à attirer les filles de familles dominantes. Nous pensons à l'éloignement de la ville qui a pu influencer l'option des jeunes filles désireuses de devenir religieuses: elle peuvent

14 Nous avons constaté qu'à l'Hôpital-Général $49.5 \%$ des religieuses sont filles de père noble. Quelques témoignages viennent justement corroborer cette assertion. Entre autres, Charlevoix affirme que la plupart des religieuses sont des filles de condition (F.-X. Charlevoix, s.j., Histoire générale de la Nouvelle-France (Paris, 1744), III : 78). Ecrivant au Ministre, en 1731, Beauharnois et Hocquart disent que la moitié des religieuses sont des filles d'officiers et le reste, de familles bourgeoises (Beauharnois et Hocquart à Maurepas, 1731, AC, C11A, 54: 30-37). A son tour, Kalm dit que la plupart d'entre elles appartiennent à des familles nobles (Peter Kalm, Travels in North America (New York, 1966), II: 454 s.). Puis, selon Bougainville, l'Hôpital-Général de Québec, en 1757, ne compterait guère que des filles de condition (Bougainville, Mémoire sur l'état de la Nouvelle-France, en 1757, RAPQ (1923-1924), 60). Enfin, ces constatations rejoignent celles de Marcel Trudel qui a étudié la communauté de l'HôpitalGénéral de Québec sous le régime militaire. L'historien affirme que, des communautés de femmes de l'époque, c'est celle de l'Hôpital-Général qui réunit le plus grand nombre de représentantes des grandes familles (Marcel Trudel, L'Eglise canadienne sous le Régime militaire, 1759-1764 (Montréal, 1956, Institut d'histoire de l'Amérique française et (Québec, 1957, Presses Universitaires), II: $301 \mathrm{~s}$.). Les résultats de notre étude, pour l'ensemble du XVIII e siècle, corroborent les conclusions qu'il dégage pour la période $1759-1764$. 
craindre les invasions et les guerres en plein cœur de Québec, mais beaucoup moins à la campagne où se situe l'Hôpital-Général. Enfin, l'attrait du nouveau - l'Hôtel-Dieu et le monastère des Ursulines sont des institutions vieilles de plus de 50 ans peut avoir exercé quelque influence.

Si les religieuses de l'Hôpital-Général sont d'origine supérieure à celle des autres communautés, y a-t-il une corrélation entre leur origine sociale et les dots qu'elles apportent?

Ce qui nous frappe d'abord, quand nous examinons les dots en Nouvelle-France, c'est leur modicité. Aucune moyenne des trois communautés étudiées n'atteint 3,000 livres, montant réglementaire fixé par l'Etat. Voilà une manifestation de la pauvreté des familles en Nouvelle-France. Qui plus est, les commentaires qui accompagnent les promesses de dots montrent bien qu'une dot de 3,000 livres et plus est de nature à suggérer un état de fortune assez élevé chez celui qui l'offre. Mais les dots supérieures à 3,000 livres ne sont pas légion.

A l'Hôpital-Général où la médiocrité des dots est encore plus prononcée, nous avons trouvé une relation entre l'origine sociale des religieuses et la condition matérielle de leur famille. Comparée aux deux autres communautés - pour l'ensemble de la période - l'importance de l'origine sociale des religieuses y est inversement proportionnelle à la valeur de la fortune familiale: près de $60 \%$ de la Communauté vient des dominants - pourcentage qui classe l'Hôpital-Général au premier rang parmi les trois communautés -, alors que ces religieuses ne donnent, en moyenne, qu'une dot d'environ 2,170 livres, c'est-à-dire une dot inférieure de 51 livres environ à la dot moyenne de l'Hôtel-Dieu et de 406 livres à celle des Ursulines. Une conclusion très nette se dégage ici: une bonne partie de la Communauté provient de familles dominantes, mais sans abondance de biens. A priori, il semblerait donc plus facile, pour une jeune fille, de se faire accepter avec une petite dot par la communauté de l'HôpitalGénéral que par les deux autres communautés. Il ne faut pas croire, toutefois, que la Communauté fût assez riche pour se passer de l'argent des dots: c'est que, probablement, il était plus facile de trouver des bienfaiteurs capables de compléter les dots. Ce qui nous amène à le croire, c'est que du temps de SaintVallier, la moyenne des dots effectivement touchées était demeurée basse: l'Evêque trouvait aisément des dons pour faire subsister la Communauté. De plus, certaines supérieures, telle Louise Soumande, profitaient parfois de leurs liens de parenté avec des hommes d'affaires importants. Il y avait sans doute 
aussi d'autres motifs pour inciter les filles de l'élite à préférer ce monastère aux autres.

Toutefois, l'augmentation considérable des dots offertes par les nouvelles professes à partir de 1740 nous laisse croire que l'évolution sociale de cette communauté a été accompagnée d'une évolution remarquable du niveau économique des familles où se faisait le recrutement. Mais, dans l'ensemble de la période, les religieuses de l'Hôpital-Général jouissent d'une origine sociale supérieure à la situation économique de leur famille.

Département d'histoire

Micheline D'Allaire, Ph.D.

Université d'Ottawa 